\title{
PHOTOGRAMMETRIC 3D RECONSTRUCTION IN MATLAB: DEVELOPMENT OF A FREE TOOL
}

\author{
A. Masiero ${ }^{\mathrm{a}, *}$ \\ ${ }^{\text {a }}$ Interdepartmental Research Center of Geomatics (CIRGEO), University of Padova, \\ Viale dell'Università 16, Legnaro (PD) 35020, Italy - \\ masiero@dei.unipd.it
}

KEY WORDS: Photogrammetry, Low Cost, Matlab, Structure from Motion, IMU

\begin{abstract}
:
This paper presents the current state of development of a free Matlab tool for photogrammetric reconstruction developed at the University of Padova, Italy. The goal of this software is mostly educational, i.e. allowing students to have a close look to the specific steps which lead to the computation of a dense point cloud. As most of recently developed photogrammetric softwares, it is based on a Structure from Motion approach. Despite being mainly motivated by educational purposes, certain implementation details are clearly inspired by recent research works, e.g. limiting the computational burden of the feature matching by determining a suboptimal set of features to be considered, using information provided by external sensors to ease the matching process.
\end{abstract}

\section{INTRODUCTION}

Thanks to its accuracy and reliability, during the last two decades aerial and terrestrial Light Detection and Ranging (LiDAR) have been considered the state of the art of surveying and digital model generation. However, the recent quick development of photogrammetric reconstruction techniques based on the Structure from Motion approach (originally developed by the computer vision community (Agarwal et al., 2010 Byröd and Aström, 2010. Hartley and Zisserman, 2003 |Ma et al., 2003)) have attracted attention by the surveying, remote sensing and geomatics research communities, making photogrammetry a very frequently considered surveying solution (recently even combined with the use of drones and/or integrated with laser scanning techniques) (Remondino et al., 2005, Masiero et al., 2017, Aicardi et al., 2016).

Ease of use of 3D reconstruction software, possibility of using cheap instrumentation (e.g. standard consumer cameras, which are usually much cheaper than laser scanners) have been key factors for the success of the method and of the related commercial and free software solutions, e.g. Agisoft PhotoScan, Pix4Dmapper, MicMac, SURE, 3DF Zephyr, VisualSFM just to cite some.

Furthermore, in certain applications the possibility of easily moving the camera on the environment (e.g. just hand-carrying the camera or mounting it on a drone) can allow the generation of clouds with a more uniform distribution of the points therein with respect to laser scanners.

This paper aims at presenting the current state of development of a free Matlab tool for photogrammetric reconstruction (implementing the Structure from Motion approach) that is being developed by the Interdepartmental Research Center of Geomatics (CIRGEO) at the University Padua, Italy. The main goal of such free tool is that of providing a software particularly well suited for educational purposes, i.e. which allows to check and validate step by step the reconstruction procedure, allowing the user to fully understand strengths and weaknesses.

Among standard functionalities, the implemented software allows the use of different feature descriptors (e.g. SIFT (Lowe,

${ }^{*}$ Corresponding author.
1999), SURF (Bay et al., 2008), BRISK (Leutenegger et al., 2011), FREAK (Alahi et al., 2012)). Self-calibration or the use of calibrated cameras have been considered (Habib and Morgan, 2003. Remondino and Fraser, 2006. Heikkila and Silven, 1997 Luhmann et al., 2015| Fraser and Stamatopoulos, 2014), while possibility of generating orthophotos is being implemented as well.

Both graphical interface and shell commands can be used to use the implemented tools. Despite being mainly developed for educational purposes, this tool also implements certain interesting functionalities which can make it of interest also for certain research purposes. For instance, since Matlab is typically not the ideal tool for efficiently deal with large amount of data, multiresolution approach and techniques for decreasing memory requirements can be considered in the implementation (Lingua et al., 2009. Martinez-Rubi et al., 2017). Integration of external information (e.g. provided by an Inertial Measurement Unit) are also allowed in order to improve tie points matching (Kurz and Benhimane, 2011, Troiani et al., 2014, Masiero and Vettore, 2016. He and Habib, 2016).

Performance, in terms of reconstruction accuracy, is compared with that of commercial software (e.g. Agisoft PhotoScan) in a case study with data acquired in the Agripolis campus of the University of Padua. Since computational burden is clearly of major interest when implementing software with a programming language as Matlab, several strategies have been implemented in order to reduce the computational time (partially reducing the reconstruction accuracy)

The developed sotware actually takes advantage of several functions already implemented in Matlab (e.g. feature extraction by means of BRISK), and of other Matlab libraries ppreviously developed by other authors, i.e. SIFT feature matching with VLFeat (Vedaldi and Fulkerson, 2008), CVLab computer vision library (Fusiello, 2013), SFMedu Structure from Motion tool (Xiao, 2014). Furthermore, a future integration with web-based view tools of 3D models will also be considered (Scianna and La Guardia, 2017). 


\section{FEATURE MATCHING BETWEEN IMAGE PAIRS}

Feature matching for the computation of a set of tie points (between each image pair), and the subsequently bundle adjustment solution of the Structure from Motion problem, is done as described in the following:

- Appearance-based feature matching

- Geometry estimation and outlier rejection

\subsection{Appearance-based feature matching}

Feature extraction and appearance based matching are mostly based on the use of built-in Matlab functions (e.g. implementing SURF, BRISK and FREAK). Furthermore, the use of VLFeat allows to simulate the behavior of SIFT as well.

At this stage, considered points are those at "salient positions", e.g. corners Fig. 1 a) and (b). Each feature is described by means of a descriptor, which summarize the appearance information of the area close to the feature (typically preserving its properties up to a set of transformations, e.g. scaling, rotation along the camera optical axis).

Matching based on the use of descriptors is typically done by using $k$ - $d$ tree methods (Beis and Lowe, 1997), which are conveniently fast. Nevertheless, exhaustive search for the best match can be done as well.

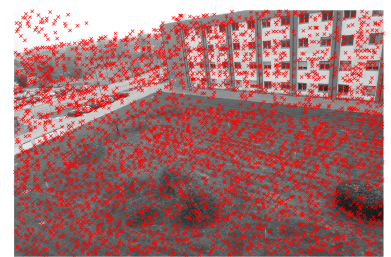

(a)

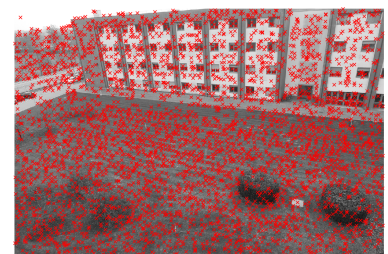

(b)
Figure 1. Feature positions for a pair of images.

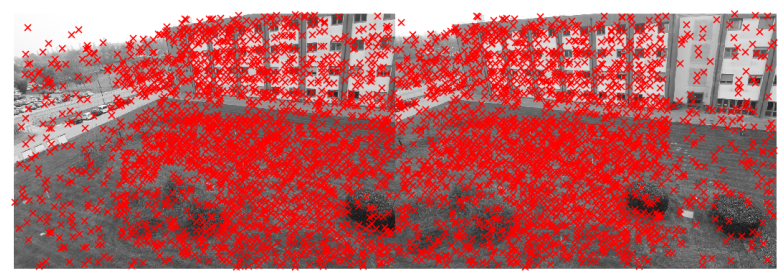

Figure 2. Feature matching based on the descriptors (appearancebased).

\subsection{Geometry estimation and outlier rejection}

Once matching is done exploiting information summarized by descriptors, geometry shall be used in order to reduce the number of wrong matches. RANdom SAmple Consensus (RANSAC) is used to estimate from the obtained matches the Fundamental matrix (Ma et al., 2003) summarizing the geometrical relation between two images.

According to the RANSAC estimation procedure, several guesses are done in order to obtain a reliable estimation of the Fundamental matrix. The developed tool allows to compare the results obtained by varying the number of guesses in the RANSAC procedure and to evaluate the variability of the obtained estimate.

Intrinsically, the RANSAC procedure provides both an estimation of the geometry between the two images and the outlier matches (i.e. to be discarded).

Fig. 3 shows the matches of Fig. 2. after outlier removal. Yellow lines in Fig. 2. link matching points in the images. The presence of mostly horizontal yellow lines ensure a quite good reliability of the obtained result.

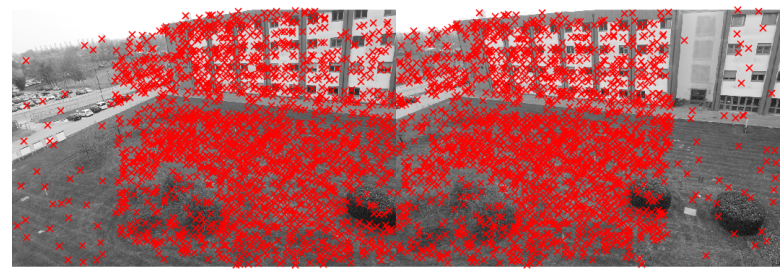

(a)

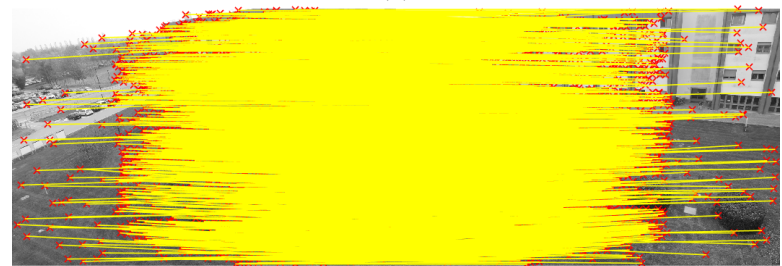

(b)

Figure 3. Feature matching (and outlier removal) based on the estimation of the epipolar geometry. (a) matches between the two images. (b) matched features linked with yellow lines.

\subsection{External information}

The recent spread of systems integrating several sensors allowed the possibility of exploiting information provided by such sensors in the matching procedure.

For instance, mobile mapping systems, based either on terrestrial/aerial vehicles or on human carried devices (Remondino et al., 2011. Al Hamad and El Sheimy, 2014, Chiang et al., 2012. Ballarin et al., 2017||Masiero et al., 2015.|Fissore et al., 2017b), which have been used in the last dozen of years in order to map and monitor areas of interest, typically integrate information provided by laser scanners, cameras with position and orientation information provided by GNSS/IMU (Inertial Measurement Unit) or other positioning sensors, e.g. (Gikas and Perakis, 2016, Goel et al., 2017).

Here integration of orientation information has been implemented as described in (Masiero and Vettore, 2016), i.e. by integrating information about orientation provide by the IMU.

Nevertheless, the integration of other strategies will be considered in the future. For instance, the use of prior information about the trajectory can be exploited as shown in (He and Habib, 2016).

\section{SPARSE RECONSTRUCTION}

Geometry between pairs of images, estimated at the previous step, is used as initial guess for the bundle adjustment procedure: Bundle adjustment is used in order to obtain a sparse reconstruction of the scene and the relative positions/orientations of the cameras. 
Motivated by recent works on the reduction of the number of tie points, in order to reduce the computational burden of this step only a subset of the original tie points is considered in the bundle adjustment. Currently the tie points to be used are randomly selected, however a more reliable strategy will be implemented in the future in the system, e.g. as described in (Martinez-Rubi et al., 2017).

\section{DENSE MATCHING}

Nowadays, semi-global matching (Hirschmuller, 2008) is widely used in order to solve the dense-matching step. Nevertheless, a simpler approach is considered in this tool in order to reduce the computational complexity, accepting a possible reduction of the accuracy: since the main goal of this tool is that of being used for educational purposes, reducing the computational burden is of fundamental importance in order to make it quickly usable by the students.

This motivated the choice of the following strategy:

- First, local interpolation on a dense set of points obtained from the sparse point cloud provides an initial estimate of dense $3 \mathrm{D}$ points.

- Each of the previously estimated points is processed: "altitude" of the point along the orthogonal direction to the local orientation of the interpolated surface is determined in order to obtain the best pixel match, in a vertical line locus (VLL) matching fashion (Linder, 2006).

In order to ease the matching procedure, similarity cost function is currently the zero mean normalized cross-correlation.

\section{RESULTS}

Fig. 4 (obtained by using Cloud Compare) compares the cloud points of the façade of a University building considered here as case study. It is worth to notice that the presented software actually was not able to completely reconstruct all the façade: areas close to the uncompleted region are those with larger difference with respect to PhotoScan reconstruction.

Agisoft PhotoScan and the presented software provided point clouds with similar cardinality of the façade (4-5 Millions of points). According to Cloud Compare comparison, average distance between the two point clouds is approximately $12 \mathrm{~cm}$.

Despite the reconstruction obtained with the proposed software is less regular than that of PhotoScan, it was also obtained by requiring much less computational burden (by a factor 10, approximately).

\section{CONCLUSIONS AND FUTURE WORKS}

This paper presented the current status of development of a photogrammetric $3 \mathrm{D}$ reconstruction tool that is under development at the University of Padova.

The presented tool will be improved in our future work in order to integrate other functionalities:

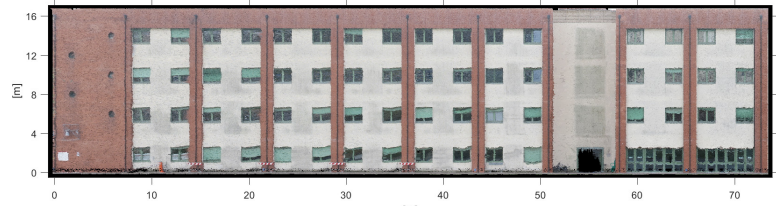

(a)

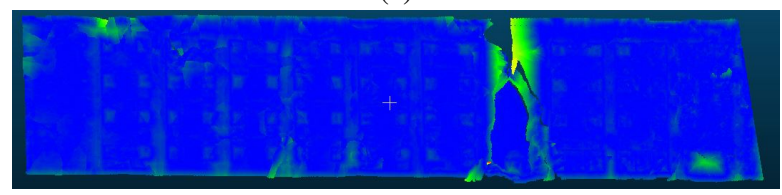

(b)

Figure 4. (a) Orthophoto of the building façade considered as case study. (b) Comparison between cloud points generated by Agisoft PhotoScan and the presented Matlab tool.

- In order to reduce time to solve bundle adjustment tie points are currently randomly subsampled. A smarter rule for tie points selection shall be implemented to reduce the estimation error, for instance similarly to the method suggested in (Martinez-Rubi et al., 2017).

- Implementation of all the functionalities shall be completed as soon as possible.

- The software has been developed to mainly deal with airborne photogrammetry (or for different cases but in similar working conditions, e.g. (Fissore et al., 2017a)). However, its extension and validation on a larger variety of cases shall be considered, for instance related also to the reconstruction of closed shapes (e.g. as statues), which is quite common in cultural heritage applications (Costantino and Angelini, 2012, Faresin et al., 2012, Kersten et al., 2004 Spangher et al., 2017).

- Despite Matlab is widely used, it is a commercial product. The development of this tool in a free Matlab-like programming language shall be considered in the future.

Once completed the main parts of the software it will be available through the website of the University of Padova.

\section{REFERENCES}

Agarwal, S., Snavely, N., Seitz, S. and Szeliski, R., 2010. Bundle adjustment in the large. In: European Conference on Computer Vision, Lecture Notes in Computer Science, Vol. 6312, pp. 2942 .

Aicardi, I., Dabove, P., Lingua, A. M. and Piras, M., 2016. Integration between TLS and UAV photogrammetry techniques for forestry applications. iForest-Biogeosciences and Forestry 10(1), pp. 41.

Al Hamad, A. and El Sheimy, N., 2014. Smartphone based mobile mapping systems. ISPRS - International Archives of the Photogrammetry, Remote Sensing and Spatial Information Sciences XL-5, pp. 29-34.

Alahi, A., Ortiz, R. and Vandergheynst, P., 2012. FREAK: Fast retina keypoint. In: Computer Vision and Pattern Recognition (CVPR), 2012 IEEE Conference on, IEEE, pp. 510-517. 
Ballarin, M., Balletti, C., Faccio, P., Guerra, F., Saetta, A. and Vernier, P., 2017. Survey methods for seismic vulnerability assessment of historical masonry buildings. ISPRS - International Archives of the Photogrammetry, Remote Sensing and Spatial Information Sciences XLII-5/W1, pp. 55-59.

Bay, H., Ess, A., Tuytelaars, T. and Van Gool, L., 2008. Speededup robust features (SURF). Computer vision and image understanding 110(3), pp. 346-359.

Beis, J. S. and Lowe, D. G., 1997. Shape indexing using approximate nearest-neighbour search in high-dimensional spaces. In: Computer Vision and Pattern Recognition, 1997. Proceedings., 1997 IEEE Computer Society Conference on, IEEE, pp. 1000 1006.

Byröd, M. and Aström, K., 2010. Conjugate gradient bundle adjustment. In: European Conference on Computer Vision, Lecture Notes in Computer Science, Vol. 6312, pp. 114-127.

Chiang, K.-W., Tsai, M.-L. and Chu, C.-H., 2012. The development of an UAV borne direct georeferenced photogrammetric platform for ground control point free applications. Sensors 12(7), pp. 9161-9180.

Costantino, D. and Angelini, M., 2012. Process modeling and photogrammetric production for structural investigations concerning to the collapse of palazzo edilizia in Salerno (Italy). Lecture Notes in Computer Science (including subseries Lecture Notes in Artificial Intelligence and Lecture Notes in Bioinformatics) 7616 LNCS, pp. 440-448.

Faresin, E., Baschiera, A., Salemi, G., Asolati, M. and Crisafulli, C., 2012. Micrometer multiresolution laser scanning of a renaissance medallion. Lecture Notes in Computer Science (including subseries Lecture Notes in Artificial Intelligence and Lecture Notes in Bioinformatics) 7616 LNCS, pp. 525-532.

Fissore, F., Guarnieri, A. and Vettore, A., 2017a. Digital model of walls of padua low relief. ISPRS - International Archives of the Photogrammetry, Remote Sensing and Spatial Information Sciences.

Fissore, F., Pirotti, F. and Vettore, A., 2017b. Open source web tool for tracking in a low cost MMS. ISPRS - International Archives of the Photogrammetry, Remote Sensing and Spatial Information Sciences.

Fraser, C. and Stamatopoulos, C., 2014. Automated target-free camera calibration. In: Proceedings of the ASPRS 2014 Annual Conference (Louisville, KY, USA), Vol. 2328.

Fusiello, A., 2013. CVLab, Matlab computer vision library. http://www.diegm.uniud.it/fusiello/teaching/ visione/libro/ Accessed: 2017-10-19.

Gikas, V. and Perakis, H., 2016. Rigorous performance evaluation of smartphone GNSS/IMU sensors for its applications. Sensors $16(8)$, pp. 1240.

Goel, S., Kealy, A., Gikas, V., Retscher, G., Toth, C., Brzezinska, D.-G. and Lohani, B., 2017. Cooperative localization of unmanned aerial vehicles using GNSS, MEMS inertial, and UWB sensors. Journal of Surveying Engineering 143(4), pp. 04017007.

Habib, A. and Morgan, M., 2003. Automatic calibration of lowcost digital cameras. Optical Engineering 42(4), pp. 948-955.

Hartley, R. and Zisserman, A., 2003. Multiple View Geometry in Computer Vision. Cambridge University Press.

He, F. and Habib, A., 2016. Automated relative orientation of UAV-based imagery in the presence of prior information for the flight trajectory. Photogrammetric Engineering \& Remote Sensing 82(11), pp. 879-891.
Heikkila, J. and Silven, O., 1997. A four-step camera calibration procedure with implicit image correction. In: Computer Vision and Pattern Recognition, 1997. Proceedings., 1997 IEEE Computer Society Conference on, pp. 1106-1112.

Hirschmuller, H., 2008. Stereo processing by semiglobal matching and mutual information. IEEE Transactions on pattern analysis and machine intelligence 30(2), pp. 328-341.

Kersten, T., Pardo, C. A. and Lindstaedt, M., 2004. 3D acquisition, modelling and visualization of north german castles by digital architectural photogrammetry. ISPRS - International Archives of Photogrammetry, Remote Sensing and Spatial Information Sciences 35(Part B2), pp. 126-132.

Kurz, D. and Benhimane, S., 2011. Gravity-aware handheld augmented reality. In: Mixed and Augmented Reality (ISMAR), 2011 10th IEEE International Symposium on, IEEE, pp. 111120.

Leutenegger, S., Chli, M. and Siegwart, R. Y., 2011. BRISK: Binary robust invariant scalable keypoints. In: Computer Vision (ICCV), 2011 IEEE International Conference on, IEEE, pp. 2548-2555.

Linder, W., 2006. Digital Photogrammetry - A Practical Course. Springer-Verlag Berlin Heidelberge.

Lingua, A., Marenchino, D. and Nex, F., 2009. Performance analysis of the SIFT operator for automatic feature extraction and matching in photogrammetric applications. Sensors 9(5), pp. 3745-3766.

Lowe, D., 1999. Object recognition from local scale-invariant features. In: Computer Vision, 1999. The Proceedings of the Seventh IEEE International Conference on, Vol. 2, pp. 1150-1157 vol.2.

Luhmann, T., Fraser, C. and Maas, H.-G., 2015. Sensor modelling and camera calibration for close-range photogrammetry. ISPRS Journal of Photogrammetry and Remote Sensing 115, pp. 37-46.

Ma, Y., Soatto, S., Košecká, J. and Sastry, S., 2003. An Invitation to 3D Vision. Springer.

Martinez-Rubi, O., Nex, F., Pierrot-Deseilligny, M. and Rupnik, E., 2017. Improving FOSS photogrammetric workflows for processing large image datasets. Open Geospatial Data, Software and Standards 2(1), pp. 12.

Masiero, A. and Vettore, A., 2016. Improved feature matching for mobile devices with IMU. Sensors 16(8), pp. 1243.

Masiero, A., Fissore, F. and Vettore, A., 2017. A low cost UWB based solution for direct georeferencing UAV photogrammetry. Remote Sensing 9(5), pp. 414.

Masiero, A., Fissore, F., Guarnieri, A., Pirotti, F. and Vettore, A., 2015. UAV positioning and collision avoidance based on RSS measurements. ISPRS - International Archives of Photogrammetry, Remote Sensing and Spatial Information Sciences 40(1), pp. 219.

Remondino, F. and Fraser, C., 2006. Digital camera calibration methods: considerations and comparisons. ISPRS - International Archives of the Photogrammetry, Remote Sensing and Spatial Information Sciences XXXVI-5, pp. 266-272.

Remondino, F., Barazzetti, L., Nex, F., Scaioni, M. and Sarazzi, D., 2011. UAV photogrammetry for mapping and 3D modelingcurrent status and future perspectives. ISPRS - International Archives of the Photogrammetry, Remote Sensing and Spatial Information Sciences 38(1), pp. C22. 
Remondino, F., Guarnieri, A. and Vettore, A., 2005. 3D modeling of close-range objects: photogrammetry or laser scanning? In: Electronic Imaging 2005, International Society for Optics and Photonics, pp. 216-225.

Scianna, A. and La Guardia, M., 2017. Main features of a 3d gis for a monumental complex with an historical-cultural relevance. ISPRS - International Archives of the Photogrammetry, Remote Sensing \& Spatial Information Sciences.

Spangher, A., Visintini, D., Tucci, G. and Bonora, V., 2017. Geomatic 3D modeling of a statue (also) for structural analysis and risk evaluation: The example of San Giovannino Martelli in Florence. ISPRS - International Archives of the Photogrammetry, Remote Sensing \& Spatial Information Sciences.

Troiani, C., Martinelli, A., Laugier, C. and Scaramuzza, D., 2014. 2-point-based outlier rejection for camera-IMU systems with applications to micro aerial vehicles. In: 2014 IEEE International Conference on Robotics and Automation (ICRA), IEEE, pp. 5530-5536.

Vedaldi, A. and Fulkerson, B., 2008. VLFeat: An open and portable library of computer vision algorithms. http://www. vlfeat.org/

Xiao, J., 2014. SFMedu: A structure from motion system for education. http://vision.princeton.edu/courses/SFMedu/ 